\title{
Cohomology of toric bundles
}

\author{
P. Sankaran and V. Uma
}

\begin{abstract}
Let $p: E \longrightarrow B$ be a principal bundle with fibre and structure group the torus $T \cong$ $\left(\mathbb{C}^{*}\right)^{n}$ over a topological space $B$. Let $X$ be a nonsingular projective $T$-toric variety. One has the $X$-bundle $\pi: E(X) \longrightarrow B$ where $E(X)=E \times_{T} X, \pi([e, x])=p(e)$. This is a Zariski locally trivial fibre bundle in case $p: E \longrightarrow B$ is algebraic. The purpose of this note is to describe (i) the singular cohomology ring of $E(X)$ as an $H^{*}(B ; \mathbb{Z})$-algebra, (ii) the topological K-ring of $K^{*}(E(X))$ as a $K^{*}(B)$-algebra when $B$ is compact. When $p: E \longrightarrow B$ is algebraic over an irreducible, nonsingular, noetherian scheme over $\mathbb{C}$, we describe (iii) the Chow ring of $A^{*}(E(X))$ as an $A^{*}(B)$-algebra, and (iv) the Grothendieck ring $\mathcal{K}^{0}(E(X))$ of algebraic vector bundles on $E(X)$ as a $\mathcal{K}^{0}(B)$-algebra.
\end{abstract}

Mathematics Subject Classification (2000). 14M25, 14F15.

Keywords. Toric varieties, toric bundles, singular cohomology, Chow ring, and K-theory.

\section{Introduction}

Let $T \cong\left(\mathbb{C}^{*}\right)^{n}$ denote the complex algebraic torus. Let $M=\operatorname{Hom}_{\text {alg }}\left(T, \mathbb{C}^{*}\right) \cong \mathbb{Z}^{n}$ and $N=\operatorname{Hom}_{\text {alg }}\left(\mathbb{C}^{*}, T\right) \cong \mathbb{Z}^{n}$ denote the group of characters and the group of 1-parameter subgroups of $T$ respectively. Note that $M=N^{\vee}:=\operatorname{Hom}(N ; \mathbb{Z})$ under the natural pairing $\langle\rangle:, M \times N \longrightarrow \mathbb{Z}$, given by $\chi^{u} \circ \lambda_{v}(z)=z^{\langle u, v\rangle}$ for all $z \in \mathbb{C}^{*}$. (Here $\chi^{u} \in \mathbb{C}(T)=\mathbb{C}[M]$ denotes the character corresponding to $u \in M$ and $\lambda_{v}$ the 1-parameter subgroup corresponding to $v \in N$.)

Let $\Delta$ be a fan in $N$ such that the $T$-toric variety $X:=X(\Delta)$ is complete and non-singular. Let $p: E \longrightarrow B$ be a principal bundle with structure group and fibre the torus $T$ over an arbitrary topological space $B$. When the bundle $p: E \longrightarrow B$ is algebraic, it is well-known that the bundle $E \longrightarrow B$ is Zariski locally trivial.

Consider the fibre bundle $\pi: E(X) \longrightarrow B$ with fibre the toric variety $X$, where $E(X)$ is the fibre product $E \times_{T} X$, and the projection map is defined as $\pi([e, x])=$ $p(e)$. Note that the bundle $\pi: E(X) \longrightarrow B$ is Zariski locally trivial when $p: E \longrightarrow B$ is algebraic. In this paper, we describe the integral singular cohomology ring $H^{*}(E(X) ; \mathbb{Z})$, and the $K$-ring $K(E(X))$ when $B$ is a compact topological space. Also, when $p: E \longrightarrow B$ is algebraic and $B$ an irreducible nonsingular noetherian 
scheme over $\mathbb{C}$, we describe the Chow ring $A^{*}(E(X))$, and the Grothendieck ring $\mathcal{K}^{0}(E(X))$ of algebraic vector bundles of the complex variety $E(X)$.

Suppose that $q: V \longrightarrow X$ is a $T$-equivariant vector bundle over $X$, then we obtain a vector bundle $E(V)$ over $E(X)$ with total space $E \times_{T} V$ where the bundle projection is the map $[e, v] \mapsto[e, q(v)]$. In case $V$ is a $T$-equivariant line bundle associated to a character $\chi^{u}: T \longrightarrow \mathbb{C}^{*}$, the bundle $E(V)$ is isomorphic to the pull-back bundle $\pi^{*}\left(\xi_{u}\right)$ where $\xi_{u} \longrightarrow B$ is the line bundle got from $E \longrightarrow B$ by 'extending' the structure group via $\chi^{u}$. After fixing an isomorphism, $T \cong\left(\mathbb{C}^{*}\right)^{n}$, $u \in M$ corresponds to an element $\left(a_{1}, \cdots, a_{n}\right) \in \mathbb{Z}^{n}$. The bundle $E$ is then the principal bundle associated to the Whitney sum of line bundles $\xi_{i}, 1 \leq i \leq n$, and $E(V)$ can then be identified with the tensor product $\xi_{1}^{a_{1}} \otimes \cdots \otimes \xi_{n}^{a_{n}}$. (Here it is understood that, when $a<0, \xi^{a}=\left(\xi^{\vee}\right)^{-a}$.)

When $B$ is a non-singular variety any line bundle $\xi$ over $B$ is isomorphic to $\mathcal{O}(Y)$ for some divisor $Y$ in $B$. The divisor class $[Y]$ is the first Chern class $c_{1}(\xi) \in A^{1}(B)$ of $\xi$.

We use the notations of [12] throughout the paper.

For $k \geq 1, \Delta(k)$ will denote the set of $k$ dimensional cones in $\Delta$. We let $d=\# \Delta(1)$, and write $v_{1}, \cdots, v_{d}$ for the primitive elements of $N$ along the edges in $\Delta$. Let $\rho_{j} \in \Delta(1)$ be the edge $\mathbb{R}_{\geq 0} v_{j}$. Recall that our hypothesis that $X$ is smooth is equivalent to the statement that the set of the primitive vectors along the edges of any cone in $\Delta$ is part of a $\mathbb{Z}$-basis for $N$.

For a cone $\sigma \in \Delta, U_{\sigma}$ denotes the affine toric variety defined by $\sigma$ and $V(\sigma)$ denotes the closure in $X$ of the variety whose local equation in $U_{\sigma}$ is $\chi^{u}=0$ for all $u \notin \sigma^{\perp}, u \in \sigma^{\vee}$. The $V(\sigma), \sigma \in \Delta$, are the orbit closures for the action of $T$ on $X$.

For $1 \leq j \leq d$ let $L_{j}$ denote the $T$-equivariant line bundle over $X$ which corresponds to the piecewise linear function $\psi_{j}$ defined by $\psi_{j}\left(v_{i}\right)=-\delta_{i, j}$. The line bundle $L_{j}$ admits a global $T$-equivariant section $s_{j}$ whose zero locus is the variety $V\left(\rho_{j}\right)$.

Let $\sigma_{1}, \cdots, \sigma_{m}$ be an ordering of the cones in $\Delta(n)$. Let $\tau_{i} \in \Delta$ be the intersection with $\sigma_{i}$ of those cones $\sigma_{j}, j>i$, such that $\operatorname{dim}\left(\sigma_{i} \cap \sigma_{j}\right)=n-1$. Thus $\tau_{1}=0$, and $\tau_{m}=\sigma_{m}$. Consider the condition:

$$
\tau_{i}<\sigma_{j} \Longrightarrow i \leq j
$$

Set $\tau_{i}^{\prime}<\sigma_{i}$ to be the cone such that $\tau_{i} \cap \tau_{i}^{\prime}=0, \operatorname{dim}\left(\tau_{i}\right)+\operatorname{dim}\left(\tau_{i}^{\prime}\right)=n, 1 \leq i \leq m$. Also consider the condition

$$
\tau_{i}^{\prime}<\sigma_{j} \Longrightarrow j \leq i
$$

Note that $\tau_{i}^{\prime}$ is the intersection with $\sigma_{i}$ of those cones $\sigma_{j}$ with $j<i$ and $\operatorname{dim}\left(\sigma_{i}\right) \cap$ $\operatorname{dim}\left(\sigma_{j}\right)=n-1$ and so condition $\left(*^{\prime}\right)$ is the same as $(*)$ for the reverse ordering on $\Delta$. It is well known that when $X$ is (nonsingular) projective, then there exists an ordering of the cones in $\Delta(n)$ such that both conditions $(*)$ and $\left(*^{\prime}\right)$ hold. We shall assume that there exists an ordering of $\Delta(n)$ such that property $(*)$ holds. (See [12], §5.2.) 
By relabelling the $v_{j}$ 's if necessary, we assume that $v_{1}, \cdots, v_{n} \in N$ are primitive vectors along the edges of $\sigma_{m}$ and let $u_{1}, \cdots, u_{n}$ be the dual basis of $M$.

Definition 1.1. Let $S$ be a ring with 1 . Let $r_{i}, 1 \leq i \leq n$, be in the centre of $S$. Consider the polynomial algebra $S\left[x_{1}, \cdots, x_{d}\right]$. We denote by $I_{S}$ the two-sided ideal generated by the following two types of elements

$$
x_{j_{1}} \cdots x_{j_{k}}, 1 \leq j_{p} \leq d,
$$

where $v_{j_{1}}, \cdots, v_{j_{k}}$ do not span a cone of $\Delta$, and,

$$
y_{i}:=\sum_{1 \leq j \leq d}\left\langle u_{i}, v_{j}\right\rangle x_{j}-r_{i}, 1 \leq i \leq n .
$$

Denote by $\mathcal{I}_{S}$ the two-sided ideal generated by elements of type (i) above and the elements

$$
z_{i}:=\prod_{j,\left\langle u_{i}, v_{j}\right\rangle>0}\left(1-x_{j}\right)^{\left\langle u_{i}, v_{j}\right\rangle}-r_{i} \prod_{j,\left\langle u_{i}, v_{j}\right\rangle<0}\left(1-x_{j}\right)^{-\left\langle u_{i}, v_{j}\right\rangle}, 1 \leq i \leq n .
$$

Define $R=R(S, \Delta)=S\left[x_{1}, \cdots, x_{d}\right] / I_{S}$ and $\mathcal{R}=\mathcal{R}(S, \Delta)=S\left[x_{1}, \cdots, x_{d}\right] / \mathcal{I}_{S}$.

Note that the $S$-algebras $R$ and $\mathcal{R}$ depend not only on the fan $\Delta$, but also on the the isomorphism $N \cong \mathbb{Z}^{n}$ resulting from the choice of $\sigma_{m} \in \Delta$ and the elements $r_{i} \in S$. The only non-commutative $\operatorname{ring} S$ we need to consider is the integral cohomology ring of $B$.

Note that for any cohomology theory $\mathcal{H}, \mathcal{H}^{*}(E(X))$ is an $\mathcal{H}^{*}(B)$-algebra via the induced map $\pi^{*}: \mathcal{H}^{*}(B) \longrightarrow \mathcal{H}^{*}(E(X))$. The following is our main theorem:

Theorem 1.2. Let $\pi: E \longrightarrow B$ be a principal T-bundle over an arbitrary topological space $B$. Assume that $X$ is a smooth complete $T$-toric variety and that $\Delta(n)$ has been ordered so that $(*)$ holds. With above notations,

(i) The singular cohomology ring of $E(X)$ is isomorphic as an $H^{*}(B ; \mathbb{Z})$-algebra to $R\left(H^{*}(B ; \mathbb{Z}), \Delta\right)$, with $r_{i}=c_{1}\left(\xi_{i}^{\vee}\right) \in H^{2}(B ; \mathbb{Z})$.

(ii) When $B$ is compact, the $K$-ring $K^{*}(E(X))$ of complex vector bundles over $E(X)$ is isomorphic as a $K^{*}(B)$-algebra to $\mathcal{R}\left(K^{*}(B) ; \Delta\right)$ where $r_{i}=\left[\xi_{i}^{\vee}\right] \in K(B)$, $1 \leq i \leq n$.

Suppose $p: E \longrightarrow B$ is algebraic where $B$ irreducible, non-singular and noetherian over $\mathbb{C}$. Furthermore, assume that $\left(*^{\prime}\right)$ also holds. Then:

(iii) The Chow ring $A^{*}(E(X))$ of $E(X)$ is isomorphic as an $A^{*}(B)$-algebra to $R\left(A^{*}(B), \Delta\right)$ where $r_{i}=c_{1}\left(\xi_{i}^{\vee}\right) \in A^{1}(B), 1 \leq i \leq n$.

(iv) The ring $\mathcal{K}(E(X))$ is isomorphic as a $\mathcal{K}(B)$-algebra to $\mathcal{R}(\mathcal{K}(B), \Delta)$ where $r_{i}=\left[\xi_{i}^{\vee}\right] \in \mathcal{K}(B)$.

We shall now briefly explain the method of proof. For the first three parts, we shall use a Leray-Hirsch type theorem to obtain the structure of $\mathcal{H}^{*}(E(X))$ as a module over $\mathcal{H}^{*}(B)$. Then we shall construct a $\mathcal{H}^{*}(B)$-algebra homomorphism 
from the expected $\mathcal{H}^{*}(B)$-algebra to $\mathcal{H}^{*}(E(X))$ and verify that this algebra homomorphism is an isomorphism of $\mathcal{H}^{*}(B)$-modules. The "Leray-Hirsch" theorem we need in the context of Chow rings is due to D. Edidin and W. Graham [10]. However we give a proof which is more suited to our specific situation. (See also [11].) The "Leray-Hirsch" in the context of $K$-theory of complex vector bundles that we need is Theorem 2.7.8, [5]. For part (iv) we use a result of Grothendieck [7] to prove the analogue of Leray-Hirsch theorem.

We do not know if parts (iii) and (iv) of the main theorem remain valid without the hypothesis that $\left(*^{\prime}\right)$ hold. Neither do we know of an example where $\Delta(n)$ admits an ordering satisfying $(*)$ but no ordering that satisfies both $(*)$ and $\left(*^{\prime}\right)$. However, there are complete nonsingular varieties $X(\Delta)$ which are not projective such that $\Delta(n)$ admits an ordering satisfying both $(*)$ and $\left(*^{\prime}\right)$. The example of a complete non projective toric variety given in p. 84 [15] is seen to be one such.

Examples of algebraic bundles $E(X) \longrightarrow B$ we consider include as special cases the toric fibre bundles considered on p. 41, [12].

We were motivated by the work of Al Amrani [2] who has computed the singular cohomology ring of a weighted projective space bundle. Another motivation for us was the work of H. Pittie and A. Ram [16] who established the Pieri-Chevalley formula in $K$-theory in the context of an algberaic $G / B$ bundle associated to a principal $B$ bundle where $G$ is a complex simple algebraic group and $B$ a Borel subgroup.

\section{The rings $R$ and $\mathcal{R}$}

In this section we prove certain facts about the rings $R$ and $\mathcal{R}$ which will be needed in the proof of the main theorem.

We keep the notations of $\S 1$. We assume that $\Delta(n)$ has been so ordered that property $(*)$ holds. Recall that $v_{1}, \cdots, v_{d}$ are the primitive vectors along the edges of $\Delta$, that $v_{1}, \cdots, v_{n}$ are in $\sigma_{m}$, and that $u_{1}, \cdots, u_{n}$ is the dual basis of $M$.

For any cone $\gamma \in \Delta$, denote by $x(\gamma)$ the monomial $x_{j_{1}} \cdots x_{j_{r}} \in S\left[x_{1}, \cdots, x_{d}\right]$ where $v_{j_{1}} \cdots, v_{j_{r}}$ are the primitive vectors along the edges of $\gamma$.

Recall from $\S 1$ the definition of the $S$-algebras $R$ and $\mathcal{R}$. We shall denote by the same symbol $x(\gamma)$, in $R$ and $\mathcal{R}$, the image of the monomial $x(\gamma) \in S\left[x_{1}, \cdots, x_{d}\right]$ under the canonical quotient map.

Lemma 2.1. (i) For $u=\sum_{1 \leq i \leq n} a_{i} u_{i} \in M$, the following equality holds in $R$.

$$
\sum_{1 \leq j \leq d}\left\langle u, v_{j}\right\rangle x_{j}=r_{u}
$$

where $r_{u}=\sum_{1 \leq i \leq n} a_{i} r_{i}$. 
(ii) If $\gamma \in \Delta(r)$ is spanned by $v_{j_{1}}, v_{j_{2}}, \cdots, v_{j_{r}}$, then

$$
x_{j_{1}} x(\gamma)=-\sum_{k}\left\langle u, v_{k}\right\rangle x\left(\gamma_{k}\right)+r_{u} x(\gamma)
$$

for some $u \in M$, where the sum on the right is over those cones $\gamma_{k}$ in $\Delta(r+1)$ which are spanned by primitive vectors $v_{k}, v_{j_{1}}, v_{j_{2}}, \cdots, v_{j_{r}}$.

(iii) If $\alpha<\gamma \leq \beta$ are cones in $\Delta$ then there exist cones $\gamma_{1}, \cdots, \gamma_{s} \in \Delta$ with $\alpha<\gamma_{k}$ such that the $\gamma_{k}$ are not contained in $\beta$, and

$$
x(\gamma)=\sum_{k} c_{k} x\left(\gamma_{k}\right)+c x(\alpha)
$$

for some $c, c_{k} \in S$.

(iv) The monomials $x\left(\tau_{i}\right), 1 \leq i \leq m$, span $R$ as an $S$-module.

Proof. Part (i) follows easily from linearity of the pairing $\langle$,$\rangle with respect to the$ first argument.

(ii) Suppose $\gamma \leq \sigma$ where $\sigma$ is $n$-dimensional. Let $v_{j_{1}}, \cdots, v_{j_{n}}$ be the primitive vectors which span $\sigma$ such that the first $r$ elements $\operatorname{span} \gamma$, with $j_{1}=j$. Let $u \in M$ be the dual basis element such that $\left\langle u, v_{j_{k}}\right\rangle=\delta_{j, j_{k}}$. One has the relation:

$$
x_{j_{1}}+\sum_{k \neq j_{1}}\left\langle u, v_{k}\right\rangle x_{k}-r_{u}=0 .
$$

Multiplying both sides by $x(\gamma)$, and using the type 1 relations, we get

$$
x_{j_{1}} x(\gamma)=-\sum_{k}\left\langle u, v_{k}\right\rangle x\left(\gamma_{k}\right)+r_{u} x(\gamma)
$$

where the sum on the right is over those cones $\gamma_{k}$ in $\Delta(r+1)$ which are spanned by primitive the vectors $v_{k}, v_{j_{1}} v_{j_{2}}, \cdots, v_{j_{r}}$ where $k \neq j_{1}, \cdots, j_{n}$. This proves (ii).

(iii) Suppose $v_{j_{1}}, \cdots, v_{j_{l}}$ spans $\beta \in \Delta(l)$ such that the first $r$ of these span $\alpha$ and the first $p$ of these span $\gamma, p>r$. Without loss of generality we may assume that $\beta$ is an $n$ dimensional cone so that $l=n$, and $v_{j_{1}}, \cdots, v_{j_{n}}$ is a basis for $N$. Now let $u \in M$ be the dual basis element so that $\left\langle u, v_{j_{q}}\right\rangle=\delta_{p, q}$. Then we have

$$
x_{j_{p}}+\sum_{k \neq j_{p}}\left\langle u, v_{k}\right\rangle x_{k}-r_{u}=0 .
$$

Multiplying by $x_{j_{1}} \cdots x_{j_{p-1}}$ and observing that the coefficient of $x_{k}$ in the sum is zero for $k \in\left\{j_{1}, \cdots, j_{n}\right\}$ and $k \neq j_{p}$, we get $x(\gamma)+\sum\left\langle u, v_{k}\right\rangle x\left(\gamma_{k}\right)-r_{u} x\left(\gamma^{\prime}\right)=0$ where $\gamma^{\prime}$ is the cone spanned by $v_{j_{1}}, \cdots, v_{j_{p-1}}$ and the sum is over those cones $\gamma_{k} \in \Delta(p)$ which are spanned by $v_{j_{1}}, v_{j_{2}}, \cdots, v_{j_{p-1}}, v_{k}, k \neq j_{1}, \cdots, j_{n}$. Note that each of these $\gamma_{k}$ contains $\alpha$ but is not contained in $\beta$. If $\gamma^{\prime}=\alpha$, we are done. Otherwise, by an induction on the dimension of $\gamma$ the statement is true for $\gamma^{\prime}$. Substituting this expression for $x\left(\gamma^{\prime}\right)$ in (1), we see that (iii) holds.

(iv) We first prove that the $x\left(\tau_{i}\right)$ span $R$. In view of (ii), it suffices to prove that for any $\gamma, x(\gamma)$ is in the $S$-submodule spanned by the $x\left(\tau_{i}\right)$. Property $(*)$ 
implies that given any $\gamma \in \Delta$, there exists a unique $i$ such that $\tau_{i} \leq \gamma \leq \sigma_{i}$; indeed it is the smallest $i$ for which $\gamma \leq \sigma_{i}$. (See [12], §5.2.) We prove, by a downward induction on this $i$, that $x(\gamma)$ is in the $S$-span of $x\left(\tau_{j}\right), j \geq i$. If $i=m$, then $\gamma=\sigma_{m}=\tau_{m}$ and there is nothing to prove.

Let $\tau_{i} \leq \gamma \leq \sigma_{i}$ for some $i<m$. Now, using (iii), we can write $x(\gamma)$ as an $S$-linear combination of $x\left(\tau_{i}\right)$ and $x\left(\gamma_{j}\right)$ where $\tau_{i}<\gamma_{j}$, and $\gamma_{j}$ is not contained in $\sigma_{i}$. It follows that each $\gamma_{j}$ is such that $\tau_{r} \leq \gamma_{j} \leq \sigma_{r}$ for some $r$ (depending on $j$ ) with $r>i$. By inductive hypothesis, each of the $x\left(\gamma_{j}\right)$ is in the $S$-span of $x\left(\tau_{q}\right), q \geq r$. It follows that $x(\gamma)$ is in the $S$-span of $x\left(\tau_{r}\right), r \geq i$, completing the proof.

Concerning the structure of $\mathcal{R}$ we have the following.

Lemma 2.2. Assume that the elements $r_{i} \in S, 1 \leq i \leq n$ are invertible. Then

(i) For $u=\sum_{1 \leq i \leq n} a_{i} u_{i} \in M$ the following relation holds in $\mathcal{R}$ :

$$
z_{u}:=\prod_{j,\left\langle u, v_{j}\right\rangle>0}\left(1-x_{j}\right)^{\left\langle u, v_{j}\right\rangle}-r_{u} \prod_{j,\left\langle u, v_{j}\right\rangle<0}\left(1-x_{j}\right)^{-\left\langle u, v_{j}\right\rangle}=0
$$

where $r_{u}=\prod_{1 \leq i \leq n} r_{i}^{a_{i}}$.

(ii) Let $\alpha<\gamma \leq \beta$ be cones in $\Delta$. Suppose that $\gamma$ is spanned by $v_{j_{1}}, \cdots, v_{j_{k}}$, then

$$
x_{j_{1}} x(\gamma)=\left(1-r_{u}\right) x(\gamma)+\sum_{p} a_{p} x\left(\gamma_{p}\right)
$$

where $a_{p} \in S$, and $\gamma_{p} \in \Delta$ are such that $\alpha<\gamma_{p}, \gamma_{p}$ are not contained in $\beta$ and $\operatorname{dim}\left(\gamma_{p}\right)>\operatorname{dim}(\gamma)$

(iii) Let $\alpha<\gamma \leq \beta$ be cones in $\Delta$. Then

$$
x(\gamma)=\sum_{p} b_{p} x\left(\gamma_{p}\right)+b x(\alpha)
$$

for some $b_{p}, b \in S$ and suitable cones $\gamma_{p} \in \Delta$ which contain $\alpha$ and are not contained in $\beta$.

(iv) The monomials $x\left(\tau_{i}\right), 1 \leq i \leq m$ span $\mathcal{R}$ as an $S$-module.

Proof. Proof of (i) is an easy exercise.

(ii) Without loss of generality, we may assume that $\beta$ is an $n$-dimensional cone. We prove this by descending induction on the dimension of $\gamma$. Suppose that $v_{j_{1}}, \cdots, v_{j_{n}}$ span $\beta$, and that $v_{j_{1}} \notin \alpha$. Let $u \in M$ be the dual basis element such that $\left\langle u, v_{j_{r}}\right\rangle=\delta_{1, r}$. The relation $z_{u}=0$ can be rewritten as

$$
\left(1-x_{j_{1}}\right) \prod_{j,\left\langle u, v_{j}\right\rangle>0}\left(1-x_{j}\right)^{\left\langle u, v_{j}\right\rangle}=r_{u} \prod_{j,\left\langle u, v_{j}\right\rangle<0}\left(1-x_{j}\right)^{-\left\langle u, v_{j}\right\rangle} .
$$

Note that none of the $x_{j_{r}}, 2 \leq r \leq n$ occur in the above relation. Multiplying both 
sides by $x(\gamma)$,

$$
\left(x(\gamma)-x_{j_{1}} x(\gamma)\right) \prod_{p}\left(1-x_{p}\right)^{\left\langle u, v_{p}\right\rangle}=r_{u} x(\gamma) \prod_{q}\left(1-x_{q}\right)^{-\left\langle u, v_{q}\right\rangle}
$$

where the product is over those $p$, (resp. $q$ ) such that $v_{p}$, (resp. $\left.v_{q}\right), v_{j_{1}} \cdots, v_{j_{k}}$ span a cone of $\Delta,\left\langle u, v_{p}\right\rangle>0$, (resp. $\left.\left\langle u, v_{q}\right\rangle<0\right)$. In particular, if $\gamma$ is $n$-dimensional, then the above equation reads $x_{j_{1}} x(\gamma)=\left(1-r_{u}\right) x(\gamma)$, which proves the lemma in this case. Assume that $k<n$ and that the statement holds for all higher dimensional cones. Then from equation (2), we see that the lemma follows by repeated application of the inductive hypothesis and by the observation that if $\gamma^{\prime}<\gamma^{\prime \prime}$ and if $\gamma^{\prime}$ is not contained in $\beta$, then neither is $\gamma^{\prime \prime}$.

Parts (iii) and (iv) follow from (ii) just as in the proof of Lemma 2.1.

Remark 2.3. One can show that if $r_{i}=0$ for all $i, 1 \leq i \leq n$, then $x_{j}^{n+1}=$ $0,1 \leq j \leq d$ in $R$ and that $x\left(\tau_{i}\right), 1 \leq i \leq m$, form a basis for $R$ as a module over $S$. Similarly, if $r_{i}=1$ for all $1 \leq i \leq n$, then $x_{j}^{n+1}=0$ for $1 \leq j \leq d$ and $x\left(\tau_{i}\right), 1 \leq i \leq m$ form a basis for $\mathcal{R}$ as an $S$-module.

\section{Singular cohomology and Chow ring}

In this section we shall prove parts (i) and (iii) of the Main Theorem 1.2.

Let $\Delta$ be a complete nonsingular fan in $N$. We assume that $\sigma_{1}, \cdots, \sigma_{m}$ is an ordering of $\Delta(n)$ such that property $(*)$ holds. (See $\S 1$.) This implies that the toric variety $X=X(\Delta)$ has an algebraic cell decomposition, namely, there exists closed subvarieties $X=Z_{1} \supset \cdots \supset Z_{m}$ of $X$ such that $Z_{i} \backslash Z_{i+1}=: Y_{i} \cong \mathbb{C}^{k_{i}}$ for some integers $k_{i}$. In fact, with $\tau_{i}$ as in $\S 1$, the closure of $Y_{i}$ is just the variety $V\left(\tau_{i}\right)$. See [12]. This yields the structure of a (finite) CW complex on $X$ with cells only in even dimensions.

Notation. We shall denote $V\left(\tau_{i}\right)$ by $V_{i}$. If $\left(*^{\prime}\right)$ also holds, then we set $V_{i}^{\prime}=V\left(\tau_{i}^{\prime}\right)$.

Assume that $p: E \longrightarrow B$ is complex algebraic and $B$ irreducible, nonsingular, and noetherian over $\mathbb{C}$. Now since the varieties $V_{i}$ are stable under the $T$-action, one has the associated bundles $\pi_{i}: E\left(V_{i}\right) \longrightarrow B$ with fibre $V_{i}$. Note that $E\left(V_{i}\right)$ is a smooth closed subvariety of $E(X)$. For any closed subvariety $Z$ in an algebraic variety $Y$ we denote by $[Z]$ its rational equivalence class in $A_{*}(Y)$. If $Z$ and $Y$ are smooth, we denote by $[Z]$ the cohomology class dual to $Z$ in $H^{2 r}(Y ; \mathbb{Z})$ as well as the element in the Chow cohomology group $A^{r}(Y)$ where $r$ is the codimension of $Z$ in $Y$.

In case property $\left(*^{\prime}\right)$ also holds, then $\left[V_{i}\right] \cdot\left[V_{j}^{\prime}\right]=0$ if $j<i$, and $\left[V_{i}\right]\left[V_{i}^{\prime}\right] \in$ $H^{2 n}(X ; \mathbb{Z}) \cong \mathbb{Z}$ is the positive generator with respect to the standard orientation coming from the complex structure on $X$. Also, in the Chow ring, $\left[V_{i}\right]\left[V_{i}^{\prime}\right] \in$ $A^{n}(X)=A_{0}(X) \cong \mathbb{Z}$ denotes the class of the point $\left[V\left(\sigma_{i}\right)\right]$ which generates $A_{0}(X)$. 
Lemma 3.1. Let $X$ be a complete nonsingular $T$-toric variety and suppose that property $(*)$ holds for an ordering of $\Delta(n)$. Let $\pi: E \longrightarrow B$ be a principal $T$-bundle over any topological space. Then:

(i) The bundle $\pi: E(X) \longrightarrow B$ admits a cohomology extension of the fibre in singular cohomology with integer coefficients. $H^{*}(E(X) ; \mathbb{Z})$ is isomorphic to $H^{*}(B ; \mathbb{Z}) \otimes H^{*}(X ; \mathbb{Z})$ as an $H^{*}(B ; \mathbb{Z})$-module.

Assume $\pi: E \longrightarrow B$ is complex algebraic where $B$ an irreducible, nonsingular noetherian variety over $\mathbb{C}$. Suppose that properties $(*),\left(*^{\prime}\right)$ hold. Then:

(ii) The Chow group $A^{*}(E(X))$ is isomorphic as an $A^{*}(B)$-module to $A^{*}(B) \otimes$ $A^{*}(X)$.

Proof. We shall fix a base point $b_{0} \in B$ and identify $X$ with the fibre $\pi^{-1}\left(b_{0}\right) \subset$ $E(X)$. (i). Since the $X$ has a CW decomposition with only even dimensional cells, its integral cohomology is isomorphic to the free abelian group with basis labelled by its cells. Indeed the dual cohomology classes $\left[V_{i}\right] \in H^{2 l_{i}}(X ; \mathbb{Z}), l_{i}=\operatorname{dim}\left(\tau_{i}\right)$, form a $\mathbb{Z}$-basis for $H^{*}(X ; \mathbb{Z})$.

Let $\eta \in \Delta(r)$ spanned by the primitive vectors along $v_{j_{1}}, \cdots, v_{j_{r}}$. Denote by $L(\eta)$ the $T$-equivariant bundle $L_{j_{1}} \oplus \cdots \oplus L_{j_{r}}$, where the $L_{j}$ are as defined in $\S 1$. The class $[V(\eta)] \in H^{2 r}(X ; \mathbb{Z})$ equals the the Chern class $c_{r}(L(\eta))=c_{1}\left(L_{j_{i}}\right) \cdots c_{1}\left(L_{j_{r}}\right)$. The bundle $\mathcal{L}(\eta)=E(L(\eta))$ over $E(X)$ restricts to $L(\eta)$ over $X$. By the naturality of Chern classes, $c_{r}(\mathcal{L}(\eta)) \in H^{2 r}(E(X) ; \mathbb{Z})$ restricts to $c_{r}(L(\eta))=[V(\eta)] \in$ $H^{2 r}(X ; \mathbb{Z})$. In particular, it follows that $\left[V\left(\tau_{i}\right)\right], 1 \leq i \leq m$, are in the image of the restriction homomorphism $H^{*}(E(X) ; \mathbb{Z}) \longrightarrow H^{*}(X ; \mathbb{Z})$. The lemma follows by Leray-Hirsch theorem ([17], p. 258).

(ii) Our proof follows that of Lemma 2.8 [11] closely. (See also Lemma 6, [10].) Clearly the classes $\left[E\left(V_{i}\right)\right] \in A^{*}\left(E\left(V_{i}\right)\right), 1 \leq i \leq m$ restrict to elements of a $\mathbb{Z}$ basis (namely $\left[V_{i}\right] \in A^{*}(X)$ ). Consider the $A^{*}(B)$-linear map $\Phi: A^{*}(B) \otimes$ $A^{*}(X) \longrightarrow A^{*}(E(X))$, defined as

$$
\Phi\left(\sum_{1 \leq i \leq m} b_{i} \otimes\left[V_{i}\right]\right)=\sum_{1 \leq i \leq m} \pi^{*}\left(b_{i}\right) \cdot\left[E\left(V_{i}\right)\right] .
$$

To prove (ii) we show that $\Phi$ is an isomorphism. Suppose $\Phi\left(\sum b_{i} \otimes\left[V_{j}\right]\right)=0 \in$ $A^{*}(E(X))$. Assume that $k$ is the smallest integer such that $b_{k} \neq 0$. Since for $j \geq k, V_{j}$ and $V_{k}^{\prime}$ are disjoint unless $j=k$ in which case they intersect transversally and $V_{j} \cap V_{j}^{\prime}=V\left(\sigma_{j}\right)$ scheme theoretically. We see that $\left[E\left(V_{j}\right)\right] \cdot\left[E\left(V_{k}^{\prime}\right)\right]=0$ if $j>k$, and, $E\left(V_{j}\right), E\left(V_{j}^{\prime}\right)$ intersect transversally and so $E\left(V_{j}\right) \cap E\left(V_{j}^{\prime}\right)=E\left(V\left(\sigma_{j}\right)\right)$ scheme theoretically (where the subvarieties are given the reduced scheme structure). Therefore, $\left[E\left(V_{j}\right)\right]$. $\left[E\left(V_{j}^{\prime}\right)\right]=\left[E\left(V\left(\sigma_{j}\right)\right)\right]$. Note that since $V\left(\sigma_{j}\right)$ is a $T$-fixed point, $E\left(V\left(\sigma_{j}\right)\right) \cong E / T=B$. Denote by $\pi_{j}$ the restriction of $\pi: E(X) \longrightarrow B$ to $E\left(V\left(\sigma_{j}\right)\right)$. Also let $\iota_{j}$ be the inclusion $E\left(V\left(\sigma_{j}\right)\right) \subset E(X)$. Note that $\left[E\left(V\left(\sigma_{j}\right)\right)\right]=$ $\iota_{j *} \pi_{j}^{*}([B]) \in A_{*}(E(X))$. 
Now since $\Phi\left(\sum_{1 \leq j \leq m} b_{j} \otimes\left[V_{j}\right]\right)=0$, we get

$$
\begin{aligned}
0=\left[E\left(V_{k}^{\prime}\right)\right] \cdot \Phi\left(\sum_{1 \leq j \leq m} b_{j} \otimes\left[V_{j}\right]\right) & =\sum_{1 \leq j \leq m} \pi^{*}\left(b_{j}\right)\left[E\left(V_{j}\right)\right] \cdot\left[E\left(V_{k}^{\prime}\right)\right] \\
& =\pi^{*}\left(b_{k}\right)\left[E\left(V\left(\sigma_{k}\right)\right)\right]=\pi^{*}\left(b_{k}\right) \cdot \iota_{k *} \pi_{k}^{*}([B]) .
\end{aligned}
$$

Applying $\pi_{*}$ and using the projection formula we get

$$
0=\pi_{*}\left(\pi^{*}\left(b_{k}\right) \cdot \iota_{k *} \pi_{k}^{*}([B])\right)=b_{k} \cdot \pi_{*} \iota_{k *} \pi_{k}^{*}([B])=b_{k} \cdot \pi_{k *} \pi_{k}^{*}([B])=b_{k} \cdot[B]=b_{k} .
$$

This contradicts our hypothesis that $b_{k} \neq 0$. It follows that $\Phi$ is injective.

We now prove surjectivity of $\Phi$. One has the filtration $B \cong E\left(Z_{m}\right) \subset \cdots \subset$ $E\left(Z_{1}\right)=E(X)$. We claim that $\Phi$ defines surjections

$$
\Phi_{i}: A^{*}(B) \otimes A^{*}\left(Z_{i}\right) \longrightarrow A^{*}\left(E\left(Z_{i}\right)\right)
$$

for each $i, 1 \leq i \leq m$. We prove this by downward induction on $i$. This is trivially true for $i=m$, since in this case $E\left(Z_{m}\right) \cong B$. Consider the diagram

$$
\begin{aligned}
& A^{*}(B) \otimes A^{*}\left(Z_{i+1}\right) \longrightarrow A *(B) \otimes A^{*}\left(Z_{i}\right) \longrightarrow A^{*}(B) \otimes A^{*}\left(Y_{i}\right) \longrightarrow 0 \\
& \Phi_{i+1} \downarrow \quad \Phi_{i} \downarrow \quad \downarrow \\
& A^{*}\left(E\left(Z_{i+1}\right)\right) \quad \longrightarrow \quad A^{*}\left(E\left(Z_{i}\right)\right) \quad \longrightarrow \quad A^{*}\left(E\left(Y_{i}\right)\right) \quad \longrightarrow 0
\end{aligned}
$$

where the top horizontal row is obtained from tensoring with $A^{*}(B)$ the exact sequence $A^{*}\left(Z_{i+1}\right) \longrightarrow A^{*}\left(Z_{i}\right) \longrightarrow A^{*}\left(Y_{i}\right) \longrightarrow 0$. The homomorphism $A^{*}(B) \otimes$ $A^{*}\left(Y_{i}\right) \longrightarrow A^{*}\left(E\left(Y_{i}\right)\right)$ is an isomorphism by Prop. 1.9, Ch. 1, [13]. Therefore the surjectivity of $\Phi_{i}$ follows by a diagram chase.

Remark 3.2. It follows from the proof of the above lemma that the classes $c_{1}\left(\mathcal{L}_{j}\right) \in H^{2}(E(X) ; \mathbb{Z}), 1 \leq j \leq d$, generate $H^{*}(E(X) ; \mathbb{Z})$ as an $H^{*}(B ; \mathbb{Z})$-algebra. Similarly, when $p: E \longrightarrow B$ is algebraic and $B$ a complete nonsingular variety, then $\left[E\left(V\left(\rho_{j}\right)\right] \in A^{1}(E(X)), 1 \leq j \leq d\right.$, generate $A^{*}(E(X))$ as an algebra over $A^{*}(B)$.

We now turn to the ring structure of $H^{*}(E(X) ; \mathbb{Z})$ and $A^{*}(E(X))$.

Recall from $\S 1$ that the line bundle $L_{j}$ over $X$ admits a $T$-equivariant section $s_{j}: X \longrightarrow L_{j}$ whose zero locus is the divisor $V\left(\rho_{j}\right)$.

Suppose that $v_{j_{1}}, \cdots, v_{j_{r}}$ does not span a cone in $\Delta$. Then $s=\left(s_{j_{1}}, \cdots, s_{j_{r}}\right)$ is a nowhere vanishing $T$-invariant section of $L_{j_{1}} \oplus \cdots \oplus L_{j_{r}}$. By taking associated construction, we see that the bundle $\mathcal{L}_{j_{1}} \oplus \cdots \oplus \mathcal{L}_{j_{r}}$ admits a nowhere vanishing section. This implies that

$$
c_{1}\left(\mathcal{L}_{j_{1}}\right) \cdots c_{1}\left(\mathcal{L}_{j_{r}}\right)=0
$$

in $H^{2 r}(E(X) ; \mathbb{Z})$.

When $p: E \longrightarrow B$ is algebraic with $B$ nonsingular, we see that

$$
\left[E\left(V\left(\rho_{j_{1}}\right)\right)\right] \cdots\left[E\left(V\left(\rho_{j_{r}}\right)\right)\right]=0
$$

in the Chow ring $A^{*}(E(X))$. 
Now, let $u \in M$ be any element. Consider the $T$-equivariant line bundle $L_{u}$ on $X$ corresponding to the principal divisor $\sum_{1 \leq j \leq d}\left\langle u, v_{j}\right\rangle V\left(\rho_{j}\right)=\operatorname{div}\left(\chi^{-u}\right)$. Clearly $L_{u}$ is isomorphic as a $T$-equivariant bundle to $\prod_{1 \leq j \leq d} L_{j}^{\left\langle u, v_{j}\right\rangle}$ as both of these bundles correspond to the same piecewise linear function $-u:|\Delta| \longrightarrow \mathbb{R}$. (See [12].) Hence $E\left(L_{u}\right) \cong \prod_{1 \leq j \leq d} \mathcal{L}_{j}^{\left\langle u, v_{j}\right\rangle}$. On the other hand the bundle $\mathcal{L}_{u}:=$ $E\left(L_{u}\right)=E\left(\chi^{-u}\right)$ is isomorphic to $\pi^{*}\left(\xi_{1}\right)^{a_{1}} \cdots \pi^{*}\left(\xi_{n}\right)^{a_{n}}$, where $a_{i}=\left\langle-u, v_{i}\right\rangle$. This yields the following relations:

$$
\sum_{1 \leq j \leq d}\left\langle u, v_{j}\right\rangle c_{1}\left(\mathcal{L}_{j}\right)-\sum_{1 \leq i \leq n}\left\langle u, v_{i}\right\rangle c_{1}\left(\pi^{*}\left(\xi_{i}^{\vee}\right)\right)=0
$$

in $H^{2}(E(X) ; \mathbb{Z})$. In the case when $p: E \longrightarrow B$ is algebraic and $B$ is nonsingular we obtain, in the Chow group $A^{1}(E(X))$,

$$
\sum_{1 \leq j \leq d}\left\langle u, v_{j}\right\rangle\left[E\left(V\left(\rho_{j}\right)\right)\right]-\sum_{1 \leq i \leq n}\left\langle u, v_{i}\right\rangle c_{1}\left(\pi^{*}\left(\xi_{i}^{\vee}\right)\right)=0 .
$$

Proof of Theorem 1.2 (i), (iii). We first consider part (iii). In view of equations (4) and (6) above we see that we have a well defined homomorphism of algebras: $\psi: R\left(A^{*}(B), \Delta\right) \longrightarrow A^{*}(E(X))$ defined by $\psi\left(x_{j}\right)=\left[E\left(V\left(\rho_{j}\right)\right)\right], 1 \leq j \leq d$.

Note that, by remark $3.2, \psi$ is surjective. We need only prove that $\psi$ is $1-1$. In view of Theorem $3.1, A^{*}(E(X))$ is a free $A^{*}(B)$-module with basis $\left[E\left(V\left(\tau_{i}\right)\right)\right]$, $1 \leq i \leq m$. It follows from Lemma 2.1 (iv) that $\psi$ is an isomorphism, completing the proof of 1.2 (iii).

Proof of part (i) is similar. In view of equations (3), (5) above, $x_{j} \mapsto c_{1}\left(\mathcal{L}_{j}\right)$ defines a homomorphism $R\left(H^{*}(B), \Delta\right) \longrightarrow H^{*}(E(X) ; \mathbb{Z})$ which is indeed an isomorphism by 2.1 (iv) and 3.1 .

Remark 3.3. If, instead of $\Delta$ being nonsingular, it is only assumed to be simplicial, then the toric variety $X$ is only an orbifold. In this case Lemma 3.1 holds provided we replace integral homology by rational homology and the Chow group by the rational Chow group throughout. In this case we note that $\left[V_{j}\right] \cdot\left[V_{j}^{\prime}\right]=q_{j}\left[V\left(\sigma_{j}\right)\right]$ for a rational number $q_{j}$ and $\left[V_{j}\right]\left[V_{k}^{\prime}\right]=0$ for $j>k$. Computing the integral cohomology or Chow ring when the fibre $X$ is only simplicial seems to be much more difficult. When $X$ is a weighted projective space Al Amrani [2] has computed the integral cohomology of $E(X)$ in a more general setting.

\section{K-theory}

In this section we prove parts (ii) and (iv) of the main theorem.

In view of our assumption in 1.2 (iv) that both the base space $B$ and the fibre $X$ are smooth, the Grothendieck ring $\mathcal{K}^{0}(E(X))$ of algebraic vector bundles may be identified, via the duality isomorphism, with Grothendieck ring $\mathcal{K}_{0}(E(X))$ of coherent sheaves on $E(X)$. We shall denote either of them by $\mathcal{K}(E(X)$. Also 
if a smooth variety $Y$ has an algebraic cell decomposition the forgetful map $\mathcal{K}^{0}(Y) \longrightarrow K(Y)$ is an isomorphism of rings. In particular, this holds when $X$ is a complete nonsingular toric variety satisfying property $(*)$ (see $\S 1$ ).

Although the $K$ ring of a complete toric variety has been studied earlier, we could not find in the literature its description in terms of generators and relations. We obtain such a description in Proposition 4.3 below. When $X$ is the projective space, such a description is due to Adams [1]. The case when $X$ is a weighted projective space is more recent, due to Al Amrani [3]. We refer the reader to [8], [14] for other descriptions of the $K$ ring as well as the equivariant $K$ ring of a toric variety.

We begin with the following lemma:

Lemma 4.1. Suppose $\zeta_{1}, \cdots, \zeta_{r}$ are complex line bundles over a finite $C W$ complex $Y$ which has cells only in even dimensions such that $H^{*}(Y ; \mathbb{Z})$ is generated by $c_{1}\left(\zeta_{1}\right), \cdots, c_{1}\left(\zeta_{r}\right) \in H^{2}(Y ; \mathbb{Z})$. Then the ring $K^{*}(Y)=K^{0}(Y)$ is generated as a ring by $\left[\zeta_{1}\right], \cdots,\left[\zeta_{r}\right] \in K(Y)$.

Proof. Let $f_{i}: Y \longrightarrow \mathbb{P}^{N}$ be a classifying map for the bundle $\zeta_{i}, 1 \leq i \leq r$ where $N>1 / 2(\operatorname{dim}(Y))$. Consider the map $f: Y \longrightarrow\left(\mathbb{P}^{N}\right)^{r}$ which is defined as $f(y)=\left(f_{1}(y), \cdots, f_{r}(y)\right)$. Then $f^{*}: H^{*}\left(\left(\mathbb{P}^{N}\right)^{r} ; \mathbb{Z}\right) \longrightarrow H^{*}(Y ; \mathbb{Z})$ is easily seen to be a surjection. By the naturality of the Atiyah-Hirzebruch [6] spectral sequence it follows that $f^{*}$ induces a surjection of $K$ groups $K\left(\left(\mathbb{P}^{N}\right)^{r}\right) \longrightarrow K(Y)$. Recall from [1] that $K\left(\mathbb{P}^{N}\right)=\mathbb{Z}[z] /\left\langle z^{N+1}\right\rangle$ where $z=[\omega]-1, \omega$ being the class of the tautological line bundle on $\mathbb{P}^{N}$. Hence $K\left(\left(\mathbb{P}^{N}\right)^{r}\right)=\mathbb{Z}\left[z_{1}, \cdots, z_{r}\right] /\left\langle z_{i}^{N+1}, 1 \leq i \leq r\right\rangle$. Since $f^{*}$ is ring homomorphism and since $f^{*}\left(z_{i}\right)=\left[\zeta_{i}\right]-1$, the lemma follows.

Lemma 4.2. Suppose that $Y$ is a complete nonsingular variety over $\mathbb{C}$ which has an algebraic cell decomposition and that $H^{*}(Y ; \mathbb{Z})$ is generated as a ring by $H^{2}(Y ; \mathbb{Z})$. Then there exist algebraic line bundles $\zeta_{1}, \cdots, \zeta_{k}$ over $Y$ such that $\mathcal{K}(Y)$ is generated as a ring by $\left[\zeta_{i}\right], 1 \leq i \leq k$. In particular, the forgetful map $\theta: \mathcal{K}(Y) \longrightarrow K(Y)$ is an isomorphism.

Proof. Since $Y$ has an algebraic cell decomposition, the Chow ring is isomorphic to the cohomology ring $H^{*}(Y ; \mathbb{Z})$, which is isomorphic as an abelian group to $\mathbb{Z}^{m}$ where $m$ is the number of cells in $Y$.

Since $A^{*}(Y)$ is torsion free, it follows that $\mathcal{K}(Y)$ is also torsion free. One has the "topological filtration" on $\mathcal{K}(Y)$ and $G r(\mathcal{K}(Y))$ denotes the associated graded group of $\mathcal{K}(Y)$. See 15.1.5 [13]. Since the map $\varphi: A^{*}(Y) \longrightarrow G r(\mathcal{K}(Y))$, defined as $[V] \mapsto\left[\mathcal{O}_{V}\right]$, is a surjective homomorphism of groups, it follows that $\mathcal{K}(Y)$ is a finitely generated abelian group of rank at most $m$.

Let $a_{1}, \cdots, a_{k}$ be a $\mathbb{Z}$-basis for $H^{2}(Y ; \mathbb{Z})$. Let $D_{1}, \cdots, D_{k}$ be divisors on $Y$ such that $\left[D_{i}\right] \in A^{1}(Y)$ maps to $a_{i} \in H^{2}(Y ; \mathbb{Z}), 1 \leq i \leq k$. Since the first Chern 
class of $\mathcal{O}\left(D_{i}\right)$ is $a_{i} \in H^{2}(Y ; \mathbb{Z})$, for $1 \leq i \leq m$, it follows that $\left[\mathcal{O}\left(D_{i}\right)\right] \in K(Y)$ generate $K(Y)$ as a ring. Thus, the forgetful homomorphism $\theta: \mathcal{K}(Y) \longrightarrow K(Y)$ is surjective.

Since the $K(Y)$ is a free abelian group of rank $m$, it follows that $\theta$ is an isomorphism. In particular, $\mathcal{K}(Y)$ is generated as ring by $\left[\mathcal{O}\left(D_{i}\right)\right] \in \mathcal{K}(Y), 1 \leq$ $i \leq k$.

Examples of varieties which satisfy the hypothesis of the above lemma are (complete nonsingular) toric varieties $X(\Delta)$ where $\Delta$ satisfies $(*)$, flag varieties $G / B$, where $G$ is semi simple and $B \subset G$ a Borel subgroup, and smooth Schubert varieties in $G / B$.

Our next result gives a description of the $K$-ring of $X$. We keep the notations of the introduction.

Recall the definition of $\mathcal{R}$ from $\S 1$.

Proposition 4.3. Let $X=X(\Delta)$ be a nonsingular complete toric variety where $\Delta$ satisfies the property $(*)$. The following relations hold in $\mathcal{K}(X)$ and $K(X)$ :

(i) $\left[\mathcal{O}_{V\left(\rho_{j_{1}}\right)}\right] \cdots\left[\mathcal{O}_{V\left(\rho_{j_{r}}\right)}\right]=0$ if $v_{j_{1}}, \cdots, v_{j_{r}}$ do not span a cone of $\Delta$,

(ii) $\prod_{1 \leq j \leq d}\left[L_{j}\right]^{\left\langle u, v_{j}\right\rangle}=1$,

(iii) Set $r_{i}=1 \in \mathbb{Z}, 1 \leq i \leq n$. The homomorphism of rings $\theta: \mathcal{R}=$ $\mathcal{R}(\mathbb{Z}, \Delta) \longrightarrow \mathcal{K}(X) \cong K(X)$ defnied by $x_{i} \mapsto\left[\mathcal{O}_{V\left(\rho_{i}\right)}\right]=\left(1-\left[L_{i}^{\vee}\right]\right)$ is an isomorphism.

Proof. Recall that $\left[\mathcal{O}_{Y}\right] .\left[\mathcal{O}_{Z}\right]=\left[\mathcal{O}_{Y \cap Z}\right]$ if $Y, Z$ are closed irreducible subvarieties of $X$ which meet transversally. Relation (i) follows from the fact that $V\left(\rho_{j_{1}}\right) \cap \cdots \cap V\left(\rho_{j_{r}}\right)=\emptyset$ if $v_{j_{1}}, \cdots, v_{j_{r}}$ does not span a cone of $\Delta$. Since for any $u \in M$ we have $\left\langle-u, v_{j}\right\rangle=\sum_{1 \leq p \leq d}\left\langle u, v_{p}\right\rangle \psi_{p}\left(v_{j}\right)$, it follows that one has a $T$-equivariant isomorphism of bundles $\prod_{1 \leq p \leq d} L_{p}^{\left\langle u, v_{p}\right\rangle} \cong L_{u}$, where $L_{u}$ is the line bundle corresponding to the piecewise linear function $-u:|\Delta|=N_{\mathbb{R}} \longrightarrow \mathbb{R}$. But $L_{u}$ is isomorphic to the trivial line bundle and so (ii) follows.

Now the section $s_{j}: X \longrightarrow L_{j}$ vanishes to order 1 on $V\left(\rho_{j}\right)$. Hence we have an exact sequence of coherent sheaves for $1 \leq j \leq d: 0 \longrightarrow L_{j}^{\vee} \longrightarrow \mathcal{O}_{X} \longrightarrow \mathcal{O}_{V\left(\rho_{j}\right)} \longrightarrow 0$. Thus $\left[\mathcal{O}_{V\left(\rho_{j}\right)}\right]=\left(1-\left[L_{j}^{\vee}\right]\right)$ in $\mathcal{K}(X)$, i.e., $\left(1-\left[\mathcal{O}_{V\left(\rho_{j}\right)}\right]\right)=L_{j}^{\vee}$. Hence $x_{j} \mapsto\left[\mathcal{O}_{V\left(\rho_{j}\right.}\right]$ defines a ring homomorphism $\theta: \mathcal{R} \longrightarrow \mathcal{K}(X)$. Since $\mathcal{K}(X)$ is free abelian of rank $m$ and since by Lemma $2.2 \mathcal{R}$ is generated by $m$ elements $x\left(\tau_{i}\right), 1 \leq i \leq m$, it follows that $\theta$ is an isomorphism, completing the proof.

Remark 4.4. Suppose $\eta_{1}, \cdots, \eta_{k}$ are line bundles over $Y$ such that their Whitney sum $\eta:=\oplus_{1 \leq i \leq k} \eta_{i}$ admits a nowhere vanishing section, then, applying the $\gamma^{k}$-operation, we obtain $\gamma^{k}(\eta-k)=0$. On the other hand, $\gamma^{k}([\eta]-k)=$ $\gamma^{k}\left(\oplus_{1 \leq i \leq k}\left(\left[\eta_{i}\right]-1\right)\right)=\prod\left(\left[\eta_{i}\right]-1\right)$. Hence, $\prod\left(1-\left[\eta_{i}\right]\right)=0$. Thus, one can avoid the use of the coherent sheaves in the proof of (i) above in the case of $K(X(\Delta))$ since we know that the section $s=\left(s_{j_{1}}, \cdots, s_{j_{r}}\right)$ of the bundle $\oplus_{1 \leq p \leq r} L_{j_{p}}$ is nowhere 
vanishing whenever $v_{j_{1}}, \cdots, v_{j_{r}}$ does not span a cone of $\Delta$.

Corollary 4.5. (i) The elements $\left[\mathcal{O}_{V\left(\tau_{i}\right)}\right] \in \mathcal{K}(X), 1 \leq i \leq m$, form a $\mathbb{Z}$-basis for $\mathcal{K}(X)$.

(ii) Let $L\left(\tau_{i}\right)=\prod_{j, v_{j} \in \tau_{i}} L_{j}$ for $1 \leq i \leq m$. Then $\left[L\left(\tau_{i}\right)\right], 1 \leq i \leq m$, form $a$ $\mathbb{Z}$-basis for $K(X)$.

Proof. This follows from the proof of 4.3 (iii).

Recall that $\mathcal{L}_{j}=E\left(L_{j}\right)$ is the line bundle over $E(X)$ with total space $E \times_{T} L_{j}$. Denote by $\mathcal{L}\left(\tau_{i}\right)$ the line bundle $E\left(L\left(\tau_{i}\right)\right)=\mathcal{L}_{j_{1}} \otimes \cdots \otimes \mathcal{L}_{j_{r}}$, where $v_{j_{1}}, \cdots, v_{j_{r}}$ are the primitive vectors along the edges of $\tau_{i}$. In view of Proposition 4.5 (ii), the restriction of the bundles $\mathcal{L}\left(\tau_{i}\right), 1 \leq i \leq m$, to the fibre $X$ form a $\mathbb{Z}$-basis for $K^{*}(X)=K^{0}(X)$. Hence, by Theorem 2.7.8 [5], it follows that $K(E(X))$ is a free $K^{*}(B)$-module with basis $\mathcal{L}\left(\tau_{i}\right), 1 \leq i \leq m$. Suppose $v_{j_{1}}, \cdots, v_{j_{r}}$ do not span a cone of $\Delta$. The $T$-equivariant section $s=\left(s_{j_{1}}, \cdots, s_{j_{r}}\right)$ of $L_{j_{1}} \oplus \cdots \oplus L_{j_{r}}$ is nowhere vanishing and extends to a nowhere vanishing section $E(s): E(X) \longrightarrow \mathcal{L}_{i_{1}} \oplus \cdots \oplus$ $\mathcal{L}_{i_{r}}$. Hence by remark 4.4 ,

$$
\prod_{1 \leq p \leq r}\left(1-\mathcal{L}_{j_{p}}\right)=0
$$

Now assume that $p: E \longrightarrow B$ is algebraic and $B$ irreducible, nonsingular and noetherian over $\mathbb{C}$. Since the $T$-equivariant sections $s_{j}$ are algebraic, equation (7) holds in $\mathcal{K}(E(X))$ as well.

For any $u \in M$, the $T$-equivariant isomorphism of bundles $\prod_{1 \leq j \leq d} L_{j}^{\left\langle u, v_{j}\right\rangle} \cong L_{u}$ yields an isomorphism of vector bundles $\prod_{1 \leq j \leq d} \mathcal{L}_{j}^{\left\langle u, v_{j}\right\rangle} \cong E\left(L_{u}\right)$. Since $E\left(L_{u}\right)=$ $\prod_{1 \leq i \leq n} \xi_{i}^{-\left\langle u, v_{i}\right\rangle}$, we get

$$
\prod_{1 \leq j \leq d} \mathcal{L}_{j}^{\left\langle u, v_{j}\right\rangle} \cong \pi^{*}\left(\xi_{u}^{\vee}\right)
$$

where $\xi_{u}=\prod_{1 \leq i \leq n} \xi_{i}^{\left\langle u, v_{i}\right\rangle}$.

We are now ready to prove the remaining parts of 1.2 .

Proof of Theorem 1.2 (ii), (iv). In view of equations (7) and (8), one has a welldefined homomorphism of $K(B)$-algebras $\Psi: \mathcal{R}=\mathcal{R}\left(K^{*}(B), \Delta\right) \longrightarrow K^{*}(E(X))$ defined by $x_{j} \longrightarrow\left(1-\left[\mathcal{L}_{j}\right]\right), 1 \leq j \leq d$.

Since the $x\left(\tau_{i}\right), 1 \leq i \leq m$ span $\mathcal{R}$ by Lemma 2.2 (iv) and since $K^{*}(E(X))$ is a free $K^{*}(B)$ module of rank $m$, it follows that $\Psi$ is an isomorphism, completing the proof of (ii).

Now let $B$ be an irreducible, nonsingular, noetherian variety over $\mathbb{C}$ and let $p: E \longrightarrow B$ be algebraic. Equations (7) and (8) still hold in $\mathcal{K}(E(X))$ since the equivariant sections $s_{j}$ are algebraic. Proceeding as above, we see that to 
complete the proof of $1.2(\mathrm{iv})$, we need only show that $\left[\mathcal{O}_{E\left(V_{i}\right)}\right], 1 \leq i \leq m$, form a basis for $\mathcal{K}(E(X))$ as a $\mathcal{K}(B)$-module, where $V_{i}$ stands for $V\left(\tau_{i}\right)$. Let $\Phi: \mathcal{K}(B) \otimes \mathcal{K}(X) \longrightarrow \mathcal{K}(E(X))$ be the $\mathcal{K}(B)$-linear map defined by $\sum_{1<i<m} b_{i} \otimes$ $\left[\mathcal{O}_{V_{i}}\right] \mapsto \sum_{1 \leq i \leq m} \pi^{*}\left(b_{i}\right)\left[\mathcal{O}_{E\left(V_{i}\right)}\right], 1 \leq i \leq m$. In view of 4.5 (i), we need only show that $\Phi$ is an isomorphism.

We first prove surjectivity of $\Phi$. This is proved by induction on the dimension of $B$, assuming only that $B$ is noetherian over $\mathbb{C}$. Without loss of generality we may assume that $B$ is irreducible. If $B$ is a point, then the result is obvious. Suppose that $\operatorname{dim}(B)>0$. Let $U$ be an affine open set in $B$ over which the $T$ bundle $p: E \longrightarrow B$ is trivial and let $Z=B \backslash U$ (with its reduced scheme structure). Note that $Z$ may not be irreducible but $\operatorname{dim}\left(Z_{k}\right)<\operatorname{dim}(B)$ for each irreducible component $Z_{k}$ of $Z$. By inductive hypothesis, $\mathcal{K}_{0}(Z) \otimes \mathcal{K}(X) \longrightarrow \mathcal{K}\left(\pi^{-1}(Z)\right)$ is surjective homomorphism of abelian groups. Consider the commuting diagram of abelian groups and their homomorphisms:

$$
\begin{aligned}
& \mathcal{K}_{0}(Z) \otimes \mathcal{K}(X) \longrightarrow \mathcal{K}_{0}(B) \otimes \mathcal{K}(X) \longrightarrow \mathcal{K}_{0}(U) \otimes \mathcal{K}(X) \longrightarrow 0 \\
& \mathcal{K}_{0}\left(\pi^{-1}(Z)\right) \quad \longrightarrow \quad \mathcal{K}_{0}(E(X)) \quad \longrightarrow \quad \mathcal{K}_{0}\left(\pi^{-1}(U)\right) \quad \longrightarrow 0
\end{aligned}
$$

where the horizontal rows are exact. The top horizontal row is got by tensoring with $\mathcal{K}(X)$ the exact sequence $\mathcal{K}_{0}(Z) \longrightarrow \mathcal{K}_{0}(B) \longrightarrow \mathcal{K}_{0}(U) \longrightarrow 0$. By Prop. 2.13, Ch. II, (Exp. 0-App.), p. 60, [7], the homomorphism $\mathcal{K}(U) \otimes \mathcal{K}(X) \longrightarrow \mathcal{K}\left(\pi^{-1}(U)\right)$ is surjective. It follows that the homomorphism $\Phi: \mathcal{K}_{0}(B) \otimes \mathcal{K}(X) \longrightarrow \mathcal{K}_{0}(E(X))$ is a surjection.

Now we prove that $\Phi$ is a monomorphism. Suppose $\Phi\left(\sum_{1 \leq i \leq m} b_{i}\left[\mathcal{O}_{V_{i}}\right]\right)=0$ where $b_{i}$ is non-zero for some $i$. Let $p \geq 1$ be the least so that $b_{p} \neq 0$. Then, writing $V_{p}^{\prime}$ for $V\left(\tau_{p}^{\prime}\right)$, we have

$$
\begin{aligned}
0=\left[\mathcal{O}_{E\left(V_{p}^{\prime}\right)}\right] \cdot\left(\sum_{1 \leq i \leq m} \pi^{*}\left(b_{i}\right) \cdot\left[\mathcal{O}_{E\left(V_{i}\right)}\right]\right) & =\sum_{p \leq i \leq m} \pi^{*}\left(b_{i}\right)\left[\mathcal{O}_{\left.E\left(V_{p}^{\prime}\right) \cap E\left(V_{i}\right)\right)}\right] \\
& =\pi^{*}\left(b_{p}\right)\left[\mathcal{O}_{E\left(V\left(\sigma_{p}\right)\right)}\right]
\end{aligned}
$$

since $\Delta$ satisfies property $\left(*^{\prime}\right)$.

Denote by $\pi_{p}$ the restriction of $\pi$ to $E\left(V\left(\sigma_{p}\right)\right)$ and by $\iota_{p}$ the inclusion $E\left(V\left(\sigma_{p}\right)\right)$ $\subset E(X)$. Then the homomorphism $\iota_{p *}: \mathcal{K}\left(E\left(V\left(\sigma_{p}\right)\right)\right) \longrightarrow \mathcal{K}(E(X))$ maps $\left[\mathcal{O}_{E\left(V\left(\sigma_{p}\right)\right)}\right]=1 \in \mathcal{K}\left(E\left(V\left(\sigma_{p}\right)\right)\right)$ to $\left[\mathcal{O}_{E\left(V\left(\sigma_{p}\right)\right)}\right] \in \mathcal{K}(E(X))$. Also, $\pi_{p}$ is an isomorphism of varieties. Therefore, applying $\pi_{*}$ to the expression $0=\pi^{*}\left(b_{p}\right)\left[\mathcal{O}_{E\left(V\left(\sigma_{p}\right)\right)}\right]$ and using the projection formula $(\S 15.1,[13])$ we get

$$
\begin{aligned}
0 & =\pi_{*}\left(\pi^{*}\left(b_{p}\right)\left[\mathcal{O}_{E\left(V\left(\sigma_{p}\right)\right)}\right]\right)=b_{p} . \pi_{*} \iota_{p *}\left(\left[\mathcal{O}_{E\left(V\left(\sigma_{p}\right)\right)}\right]\right) \\
& =b_{p} . \pi_{p *}\left(\left[\mathcal{O}_{E\left(V\left(\sigma_{p}\right)\right)}\right]\right)=b_{p}\left[\mathcal{O}_{B}\right]=b_{p} .
\end{aligned}
$$

This contradicts our choice of $p$. Hence we conclude that $\Phi$ is a monomorphism.

Concluding remark 4.6. Parts (iii) and (iv) of the main theorem also hold 
when the base field $\mathbb{C}$ is replaced by any algebraically closed field $k$. Namely, let $B$ be an irreducible nonsingular noetherian variety over $k$ and let $\pi: E \longrightarrow B$ be a principal $T$ bundle where $T=\operatorname{Spec}(k[M])$. Since any toric variety is defined over the integers the fan $\Delta$ defines a nonsingular complete $k$-scheme $X=X(\Delta)$. Again $E(X) \longrightarrow B$ is a Zariski locally trivial bundle with fibre $X$. Then $\mathcal{K}(E(X))$ and $A^{*}(E(X))$ are isomorphic to $\mathcal{R}$ and $R$ respectively.

\section{References}

[1] J. F. Adams, Vector fields on spheres, Annals of Mathematics 75 (1962), 603-632.

[2] A. Al Amrani, Cohomological study of weighted projective spaces, Algebraic Geometry (Ankara, 1995), 1-52, Lect. Notes in Pure Appl. Math. 193, Marcel Dekker, NY, 1997.

[3] A. Al Amrani, Complex K-theory of weighted projective spaces, Journal of Pure and Applied Algebra 93 (1994), 113-127.

[4] A. Al Amrani, A comparison between cohomology and K-theory of weighted projective spaces, Journal of Pure and Applied Algebra 93 (1994), 127-134.

[5] M. F. Atiyah, K-Theory, W. A. Benjamin, New York, NY, 1967.

[6] M. F. Atiyah and F. Hirzebruch, Vector bundles and homogeneous spaces, Proceedings of Symposia in Pure Mathematics, 3, Amer. Math. Soc., 1961.

[7] P. Berthelot, A. Grothendieck and L. Illusie, Theorie des intersections et theorem de Riemann-Roch, SGA-6, 1966/67, Lecture Notes in Mathematics 225, Springer-Verlag, Berlin, 1971.

[8] M. Brion and M. Vergne, An equivariant Riemann-Roch theorem for complete, simplicial toric varieties, J. Reine Angew. Math. 482, (1997), 67-92.

[9] V. I. Danilov, The geometry of toric varieties, Russian Mathematical Surveys 33 (1978), 97-154.

[10] D. Edidin and W. Graham, Characteristic classes in the Chow ring, Journal of Algebraic Geometry 6 (1997), 431-443.

[11] G. Ellingsrud and S. A. Stromme, On the Chow ring of a geometric quotient, Annals of Mathematics 130 (1989), 159-187.

[12] W. Fulton, Introduction to toric varieties, Annals of Mathematics Studies 131, Princeton University Press, Princeton, NJ, 1993.

[13] W. Fulton, Intersection theory, Ergebnisse der Mathematik und ihrer Grenzgebiete, Band 2, Springer-Verlag, NY, 1984

[14] R. Morelli, The K-Theory of a toric variety, Advances in Mathematics 100, (1993), 154-182.

[15] T. Oda, Convex bodies and algebraic geometry, Ergebnisse der Mathematik, 15, SpringerVerlag, 1988

[16] H. V. Pittie and A. Ram, A Pieri-Chevalley formula in the $K$-theory of a $G / B$-bundle, Electronic Research Announcements of the American Mathematical Society 5 (1999), 102107.

[17] E. Spanier, Algebraic topology, Springer-Verlag, NY.

P. Sankaran and V. Uma

Institute of Mathematical Sciences

CIT Campus, Chennai 600113

India

e-mail: sankaran@imsc.res.in

uma@imsc.res.in

(Received: December 19, 2001) 